\title{
Plantas de cobertura e qualidade química de Latossolo Amarelo sob plantio direto no cerrado maranhense
}

\author{
Sara B. Bressan' ${ }^{1}$, Júlio C. A. Nóbrega', Rafaela S. A. Nóbrega ${ }^{1}$, \\ Ronny S. Barbosa ${ }^{2} \&$ Lusiene B. Sousa ${ }^{1}$
}

\begin{abstract}
RESUMO
Objetivando-se avaliar, com este trabalho, o efeito de plantas de cobertura sobre os atributos químicos do solo da região de Balsas - Maranhão, amostras de um Latossolo Amarelo Distrófico sob as coberturas vegetais de milheto [Pennisetum americanum (L.) Leeke], braquiária (Brachiaria ruziziensis) e cerrado nativo (cerrado de porte médio), foram coletadas em três profundidades $(0-0,10 ; 0,10-0,20$ e 0,20-0,40 $\mathrm{m})$. As coberturas vegetais de milheto e de braquiária promoveram aumento do $\mathrm{pH}$ do solo e reduções nos teores de $\mathrm{Al}^{3+} \mathrm{e}$ na saturação por $\mathrm{Al}^{3+}$, em relação à área com cerrado nativo. Maior quantidade de resíduo foi verificada na superfície do solo da área sob cerrado nativo devido ao maior aporte de resíduos vegetais e à menor taxa de decomposição. Os teores de nutrientes e níveis de matéria orgânica foram mais elevados nas áreas sob cobertura de milheto e braquiária, sobretudo nas profundidades de 0-0,10 e 0,10$0,20 \mathrm{~m}$. As plantas de cobertura braquiária e milheto foram semelhantes quanto às alterações promovidas nos atributos químicos do solo.
\end{abstract}

Palavras-chave: teores de nutrientes, Pennisetum americanum (L.) Leeke, Brachiaria ruziziensis

\section{Plant cover and chemical quality of Oxisol under no-tillage in the cerrado of Maranhão State}

\begin{abstract}
Aiming to evaluate the effect of cover plants over soil chemical attributes in the region of Balsas - Maranhão, samples of Oxisol under plant cover of millet [Pennisetum americanum (L.) Leeke], brachiaria (Brachiaria ruziziensis) and native Cerrado (mid-sized), were collected in three depths (0-0.10;0.10-0.20 and 0.20$0.40 \mathrm{~m}$ ). Plant cover of millet and brachiaria promoted an increase in soil $\mathrm{pH}$ and reductions in amounts of $\mathrm{Al}^{3+}$ and in saturation by $\mathrm{Al}^{3+}$, in relation to the area with native Cerrado. Major amounts of residue were verified on soil surface under native cerrado, due to greater input of plant residue and lower decomposition rate. Amounts of nutrients and levels of organic matter were higher in areas under millet and brachiaria cover, mainly in 0-0.10 e 0.10-0.20 m. depths. Brachiaria and millet cover plants were similar in relation to alterations promoted in chemical attributes of soil.
\end{abstract}

Key words: nutrient content, Pennisetum americanum (L.) Leeke, Brachiaria ruziziensis

1 UFPI, Campus Profa. Cinobelina Elvas, BR 135 - km 03, Planalto Horizonte/Cibrazem, CEP 64.900-000, Bom Jesus, PI. Fone: (89) $3562-2535$. E- mail: sara.bonini@ceagrobrasil.com; juliocnobrega@gmail.com; rafaela.nobrega@gmail.com; lusienebarbosa@hotmail.com

${ }^{2}$ Departamento de Ciência do Solo/UNESP, Faculdade de Ciências Agrárias e Veterinárias de Jaboticabal. Via de Acesso Prof. Paulo Donato Catellane, s/n - Depto de Solos e Adubos - Sala/Porta 36, CEP 14884-900, Jaboticabal, SP. Fone: (16) 8177-8868. E-mail: rsbagronomo@gmail.com 


\section{INTRODUÇÃO}

O sistema plantio direto (SPD), tipo de manejo de solo que mantém os resíduos culturais na superfície do solo, constitui uma importante técnica para a manutenção e recuperação da capacidade produtiva do solo sob cultivo em regiões de clima tropical e subtropical, devido ao efeito que promove nos atributos físicos, químicos e biológicos dos solos (Falleiro et al., 2003; Almeida et al., 2005; Wendling et al., 2005; Lange et al., 2006; Azevedo et al., 2007; Aratani et al., 2009). Dentre as contribuições da manutenção dos resíduos culturais na superfície do solo, segundo Leite et al. (2009), está a ação direta e efetiva na redução da erosão hídrica em virtude da dissipação de energia cinética das gotas de chuva, diminuindo a desagregação das partículas do solo e o selamento superficial, além da melhoria das condições de fertilidade do solo (Sidiras \& Pavan, 1985; Pavan et al., 1986; Silva et al., 1997; Moraes, 2001; Canellas et al., 2003).

Apesar das alterações proporcionadas pelo SPD, cultivos sucessivos de uma única espécie comercial podem esgotar ou reduzir o estoque dos nutrientes no solo em virtude da exportação pela colheita e a reposição insuficiente dos mesmos por meio dos fertilizantes. Com isto, o uso de coberturas vegetais vivas no solo em áreas sob SPD após o cultivo da cultura principal vem sendo estudado com foco na produção e na qualidade da matéria seca, decomposição dos resíduos culturais, acúmulo e mineralização de nutrientes e produtividade da cultura comercial (Espíndola et al., 2006; Silva \& Menezes, 2007; Soratto \& Crusciol, 2007). Segundo Nunes et al. (2006) o emprego do SPD sobre a palhada implica no conhecimento e na definição das espécies as quais devem ter boa produção de biomassa e ser suficientemente persistentes para proteção física do solo e disponibilização de nutrientes, sobretudo nos períodos de excesso ou escassez de água, resultando em benefícios posteriores para a cultura.

Plantas de cobertura proporcionam vários benefícios aos ecossistemas agrícolas, como a diminuição da erosão do solo, o aumento da infiltração da água no solo, retenção de nutrientes, conteúdo de matéria orgânica (Battany \& Grismer, 2000; Steenwerth \& Belina, 2008) e água no solo (Odhiambo \& Bomke, 2007; Muñoz-Carpena et al., 2008). No geral, os benefícios atribuídos às plantas de cobertura sobre os atributos do solo decorrem, sobremaneira, do aumento da porosidade do solo, redução do impacto da energia da gota de chuva sobre a superfície do solo, aumento da matéria orgânica (Leite et al., 2003; Rao \& Li, 2003) e das funções microbianas no solo (Steenwerth \& Belina, 2008).

Na região do cerrado brasileiro a obtenção de resíduos vegetais para a cobertura do solo em áreas sob SPD tem sido difícil devido, dentre outros fatores, à baixa produção de fitomassana entresafra e à decomposição acelerada dos resíduos, condições em que o uso de espécies com decomposição mais lenta representa uma estratégia para aumentar a eficiência dessas coberturas na produção de resíduos sobre a superfície do solo. Segundo Bortoluzzi \& Eltz (2000) a taxa de decomposição dos resíduos vegetais é controlada pelas características qualitativas do material vegetal, principalmente pela relação $\mathrm{C} / \mathrm{N}$ e pelo teor de lignina, além de outras variáveis ligadas ao clima, em especial a temperatura do ar e precipitação pluvial (Espíndola et al., 2006), manejo dos restos culturais (Leite et al., 2009) e solo (Bortoluzzi \& Eltz, 2000; Leite et al., 2009).

No Município de Balsas, sul do Estado do Maranhão, os agricultores fazem uso do milheto, sorgo e braquiária, como plantas de cobertura em sucessão à soja; no entanto não há, para a localidade, estudo que permita verificar a real contribuição das diferentes espécies vegetais sobre a manutenção e/ou elevação dos teores de nutrientes e matéria orgânica do solo. Neste contexto objetivou-se, com este trabalho, avaliar o efeito das plantas de cobertura sobre os atributos químicos de um Latossolo Amarelo Distrófico da região do cerrado maranhense sob SPD.

\section{Material e Métodos}

O estudo foi realizado em talhões de lavouras comerciais, sob um Latossolo Amarelo Distrófico, situados no município de Loreto, MA, no povoado Ilha de Balsas ( $6^{\circ} 57^{\prime} \mathrm{S}, 45^{\circ} 10^{\prime} \mathrm{W}$ e $380 \mathrm{~m}$ de altitude). O clima da região é do tipo Aw, segundo o sistema de classificação de Köppen, com precipitação média anual de $1.049 \mathrm{~mm}$ e temperatura média de $26^{\circ} \mathrm{C}$. A estação seca ocorre entre abril e outubro e a estação chuvosa, de novembro a março (RADAMBRASIL, 1986).

Duas áreas sob cobertura vegetal foram selecionadas para estudo, uma com milheto [Pennisetum americanum (L.) Leeke] e outra com braquiária (Brachiaria ruziziensis) em sucessão à cultura da soja. A área com milheto foi implantada no ano agrícola de 1998/1999 e a com braquiária no ano agrícola de 1999/2000, ambas a partir do cerrado nativo. Nessas áreas a primeira calagem foi realizada com $5,5 \mathrm{t} \mathrm{ha}^{-1}$ de calcário dolomítico (PRNT 65\%) e a segunda em 2003, com 1,0 tha ${ }^{-1}$ de calcário dolomítico (PRNT 90\%). A adubação na linha de plantio ocorreu em todos os anos, com média de $350 \mathrm{~kg} \mathrm{ha}^{-1}$, variando entre as fórmulas 2-20-20 e 2-24-12 para cada ano, alternadamente.

Os tratamentos foram constituídos por três coberturas vegetais: milheto, braquiária e cerrado nativo (cerrado de porte médio) este utilizado como referência e três profundidades de amostragem de solo $(0-0,10 ; 0,10-0,20$ e $0,20-0,40 \mathrm{~m})$. As parcelas úteis apresentavam dimensões de $10,0 \mathrm{~m}$ de largura por $14,0 \mathrm{~m}$ de comprimento. Para cada tratamento foram estabelecidas três repetições. Em cada parcela coletou-se uma amostra composta de solo formada a partir de 15 amostras simples. As amostras foram secadas ao ar e passadas em peneiras de $2 \mathrm{~mm}$.

Para a área sob cobertura de milheto a menor produção de soja registrada foi de 28 sacas ha $^{-1}$ (devido a problemas climáticos) enquanto a maior produção foi de 66 sacas ha $^{-1}$. Sob cobertura com braquiária a menor produção de soja registrada

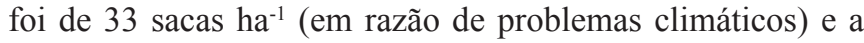
maior de 60 sacas ha $^{-1}$. A variedade de soja BRS-MT Uirapuru foi plantada no espaçamento de $0,45 \mathrm{~m}$ entre linhas e $0,10 \mathrm{~m}$ na linha.

Para avaliação da quantidade de resíduos culturais sobre a superfície do solo foram coletadas nove amostras em cada cobertura do solo utilizando-se um quadrado de madeira de $0,50 \times 0,50 \mathrm{~m}$, lançado aleatoriamente sobre a superfície do 
solo de cada área; em seguida, o material contido dentro de cada quadrado foi coletado, armazenado em sacos de papel, secado em estufa com circulação de ar, a temperatura de $65^{\circ} \mathrm{C}$ pelo período de $48 \mathrm{~h}$ e, em seguida, pesado.

Nas amostras de solo foram determinados: $\mathrm{pH}$ em água (relação solo:solução 1:2,5), teores de $\mathrm{P}, \mathrm{K}^{+} ; \mathrm{Ca}^{2+} ; \mathrm{Mg}^{2+} ; \mathrm{Al}^{3+}$, $\mathrm{Fe}^{2+}, \mathrm{Cu}^{2+}, \mathrm{Mn}^{2+}, \mathrm{Zn}^{2+}, \mathrm{H}+\mathrm{Al}$, carbono orgânico e calculados os valores de soma de bases (SB), CTC efetiva $(\mathrm{t})$ e CTC potencial $(\mathrm{T})$, saturação por bases (V\%) e por alumínio $(\mathrm{m} \%)$ de acordo com métodos compilados por EMBRAPA (1997).

$\mathrm{O}$ delineamento experimental utilizado foi o inteiramente casualisado, com estrutura em faixas, em razão das profundidades de amostragem. Os resultados foram submetidos à análise de variância e o efeito dos tratamentos comparado pelo teste de Skott-Knotta 5\% de probabilidade.Para identificar a similaridade das áreas com solos cultivados com milheto, braquiária e a de cerrado nativo, utilizou-se a estatística multivariada por meio da análise de agrupamento (análise de cluster) e componentes principais através do Software Statistica versão 7.0 (Statsoft, 2004). Inicialmente, realizou-se a análise de cluster para verificar os grupos formados no dendrograma (Figura 1). Como os dados são expressos em unidades de medidas diferentes, estes foram padronizados para assegurar que todas as variáveis contribuíram igualmente para o modelo, independente da escala.

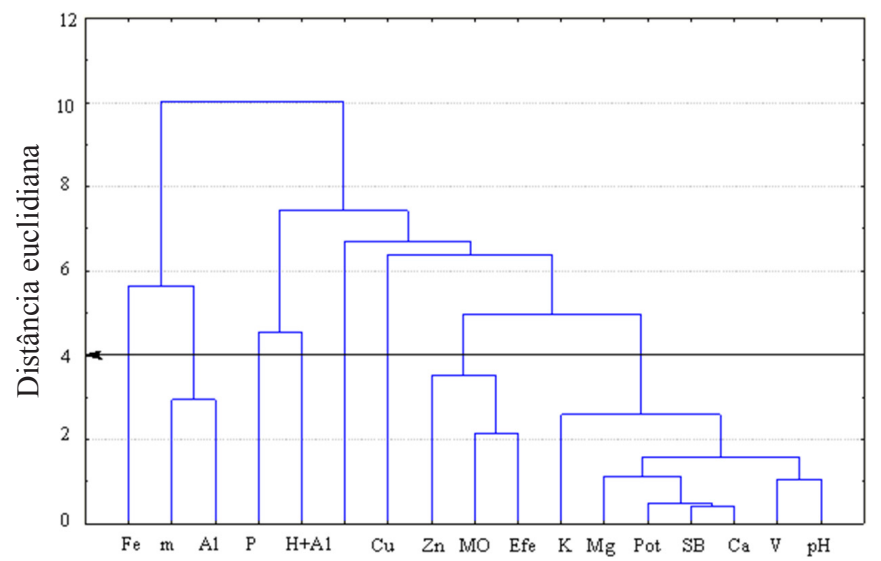

Figura 1. Dendrograma da análise de cluster (distância Euclidiana) das 17 variáveis analisadas em um Latossolo Amarelo Distrófico com diferentes plantas de cobertura sob plantio direto na região do cerrado maranhense

\section{Resultados E Discussão}

Verificou-se maior quantidade de resíduos vegetais na área sob cerrado nativo (Figura 2). Quantitativamente, um dos requisitos para o sucesso do SPD é que a camada de palhada sobre o solo seja formada por pelo menos $5.000 \mathrm{~kg} \mathrm{ha}^{-1} \mathrm{de}$ resíduos. Esta cobertura evita a ação do impacto da gota da chuva (Battany \& Grismer, 2000; Leite et al., 2009), reduz a temperatura do solo (Bortoluzzi \& Eltz, 2000) e propicia maior conteúdo de água (Odhiambo \& Bomke, 2007) e matéria orgânica no solo (Leite et al., 2003; Wendling et al., 2005; Lange et al., 2006; Steenwerth \& Belina, 2008) que, conjuntamente, melhoram os atributos químico e físico do solo (Espíndola et al.,
2006; Azevedo et al., 2007; Aratani et al., 2009) contribuindo para a estabilização da produção (Nunes et al., 2006) e recuperação ou manutenção da qualidade do solo (Azevedo et al., 2007). Neste estudo as quantidades de resíduos sobre a superfície do solo se encontram próximas e/ou superiores ao valor já citado, principalmente no caso do milheto cujo valor já se aproxima da área sob cerrado nativo. Ao avaliar o efeito de rotações de culturas no verão (milho e soja), com uso da área no inverno (feijão, milheto, mucuna, braquiária e pousio), Guimarães (2000) observou que a produção de biomassa seca das coberturas de inverno foi de 7.199,0; 7.296,0; 7.143,0; $6.912,0$ e $7.479,0 \mathrm{~kg} \mathrm{ha}^{-1}$, respectivamente.

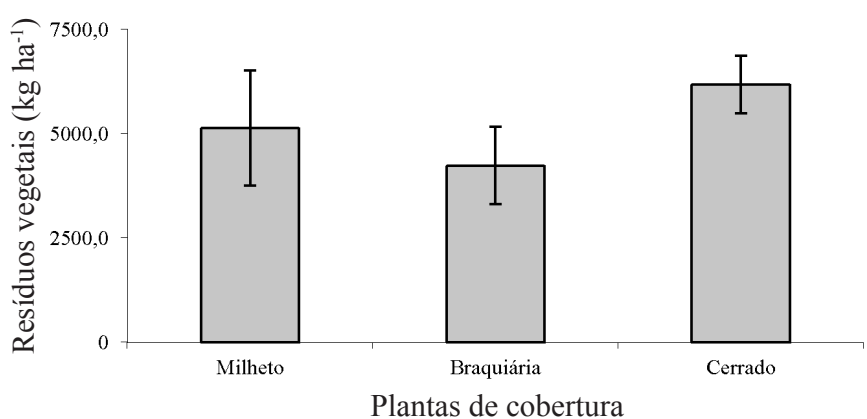

Figura 2. Produção de resíduos culturais pelas plantas de cobertura em um Latossolo Amarelo Distrófico com diferentes plantas de cobertura sob plantio direto na região do cerrado maranhense

Dentre os fatores que têm contribuído para o aporte de resíduos na área sob SPD, se destaca a decomposição mais lenta dos mesmos devido à relação $\mathrm{C} / \mathrm{N}$ mais elevada das gramíneas (Silva et al., 1997; Torres et al., 2008). Estudando a velocidade de decomposição da palhada de milheto e sorgo e o acúmulo de nutrientes na fitomassa e sua mineralização, Moraes (2001) observou que a taxa média de decomposição da palhada foi maior nos primeiros 42 dias e que a mineralização dos nutrientes é mais acentuada nos primeiros 63 dias após a dessecação e rolagem dos resíduos. Já as taxas de decomposição de crotalária, guandu, mucuna-preta e braquiária em solo sob cerrado nativo e solo descoberto, apresentaram médias de 61,3, $61,9,65,6$ e 78,9\%, respectivamente, decorridos 60 dias após a implantação das bolsas de decomposição (Silva et al., 1997).

Além da quantidade outro fator determinante na escolha da espécie de cobertura do solo para o sucesso do SPD, é a qualidade do resíduo produzido pois eleé que proporcionará maior ou menor ciclagem de nutrientes à cultura em sucessão. Para o pH (Tabela 1) houve aumento nas áreas com cobertura de milheto e braquiária em relação à área com cerrado nativo. Entre as plantas de cobertura a maior média ocorreu na área com braquiária, de forma especial na profundidade de 0 - 0,10 m. Comportamento similar ao $\mathrm{pH}$ foi observado para o $\mathrm{H}+$ $\mathrm{Al}$ mas para $\mathrm{o} \mathrm{Al}^{3+} \mathrm{e}$ saturação por $\mathrm{Al}^{3+}$ houve decréscimo nas áreas com cobertura de milheto e braquiária em relação à área com cerrado nativo.

$\mathrm{O}$ aumento do $\mathrm{pH}$ nas áreas com milheto e braquiária em referência ao cerrado nativo decorre, dentre outros aspectos, da aplicação de calcário e fertilizantes a partir de 1998/1999 para o plantio de soja, que contribui para a redução dos teores de 
Tabela 1. $\mathrm{pH}$ em água, acidez potencial $(\mathrm{H}+\mathrm{Al})$, acidez trocável $\left(\mathrm{Al}^{3+}\right)$ e saturação por alumínio em um Latossolo Amarelo Distrófico com diferentes plantas de cobertura sob plantio direto na região do cerrado maranhense

\begin{tabular}{|c|c|c|c|}
\hline \multirow{2}{*}{$\begin{array}{l}\text { Profundidade } \\
\text { (m) }\end{array}$} & \multicolumn{3}{|c|}{ Plantas de cobertura } \\
\hline & Milheto & Braquiária & Cerrado nativo \\
\hline & \multicolumn{3}{|c|}{$\mathrm{pH}$ em $\mathrm{H}_{2} \mathrm{O}(1: 2,5)$} \\
\hline $0-0,10$ & $4,33 \mathrm{bA}$ & $4,70 \mathrm{aA}$ & $3,57 \mathrm{cA}$ \\
\hline $0,10-0,20$ & $3,73 \mathrm{aB}$ & $3,67 \mathrm{aB}$ & $3,50 \mathrm{bA}$ \\
\hline \multirow[t]{2}{*}{$0,20-0,40$} & $3,50 \mathrm{aC}$ & $3,53 \mathrm{aB}$ & $3,47 \mathrm{aA}$ \\
\hline & \multicolumn{3}{|c|}{$\mathrm{Al}^{3+}\left(\mathrm{cmol}_{\mathrm{c}} \mathrm{dm}^{-3}\right)$} \\
\hline $0-0,10$ & $0,00 \mathrm{bC}$ & $0,00 \mathrm{bB}$ & $0,60 \mathrm{aA}$ \\
\hline $0,10-0,20$ & $0,53 \mathrm{bB}$ & $0,80 \mathrm{aA}$ & $0,57 \mathrm{bA}$ \\
\hline \multirow[t]{2}{*}{$0,20-0,40$} & $0,77 \mathrm{aA}$ & $0,90 \mathrm{aA}$ & $0,70 \mathrm{aA}$ \\
\hline & \multicolumn{3}{|c|}{$\mathrm{H}+\mathrm{Al}\left(\mathrm{cmol}_{\mathrm{c}} \mathrm{dm}^{-3}\right)$} \\
\hline $0-0,10$ & $6,38 \mathrm{aB}$ & $6,99 \mathrm{aC}$ & $4,51 \mathrm{bA}$ \\
\hline $0,10-0,20$ & $9,05 \mathrm{bA}$ & $13,01 \mathrm{aA}$ & $4,22 \mathrm{cA}$ \\
\hline $0,20-0,40$ & $7,26 \mathrm{bB}$ & $10,06 \mathrm{aB}$ & $3,75 \mathrm{cA}$ \\
\hline & \multicolumn{3}{|c|}{ Saturação por Al (\%) } \\
\hline $0-0,10$ & $0,00 \mathrm{bC}$ & $0,00 \mathrm{bC}$ & $47,43 \mathrm{aB}$ \\
\hline $0,10-0,20$ & $21,70 \mathrm{bB}$ & $29,10 \mathrm{bB}$ & $49,40 \mathrm{aB}$ \\
\hline $0,20-0,40$ & $47,93 \mathrm{bA}$ & $52,53 \mathrm{bA}$ & 65,57 aA \\
\hline
\end{tabular}

Médias seguidas de mesma letra minúscula na linha e maiúscula na coluna, não diferem entre si estatisticamente pelo teste de Scott-Knott a 0,05 de probabilidade

$\mathrm{Al}^{3+}$,saturação por $\mathrm{Al}^{3+} \mathrm{e}$ aumento dos teores de cátions básicos no solo; outrossim, o aumento dos níveis de matéria orgânica nas áreas sob cobertura de milheto e braquiária (Tabela 3) contribui também para a elevação do $\mathrm{pH}$ do solo e redução dos teores de $\mathrm{Al}^{3+}$. Em solos sob sistemas de café orgânico e café em conversão, Theodoro et al. (2003) identificaram a ausência de $\mathrm{Al}^{3+}$, em razão, sobremaneira, do aumento do $\mathrm{pH}$, que promove redução da solubilidade do $\mathrm{Al}^{3+}$ e provável reação de complexação do elemento por compostos orgânicos depositados no solo. Sidiras \& Pavan (1985) e Pavan et al. (1986) também verificaram que a cobertura morta de resíduos vegetais sobre a superfície do solo contribui para a elevação do $\mathrm{pH}$ e para redução do $\mathrm{Al}^{3+}$ trocável, principalmente nas camadas superficiais do solo.

Os teores de $\mathrm{P}, \mathrm{K}^{+}, \mathrm{Ca}^{2+}, \mathrm{Mg}^{2+}$ e SB (Tabela 2) nas áreas com cobertura de milheto e braquiária, foram mais elevados que na área com cerrado nativo, principalmente nas profundidades de $0-0,10$ e $0,10-0,20 \mathrm{~m}$. Dentre as coberturas vegetais a braquiária apresentou maiores médias $(\mathrm{p}<0,05)$. Os teores mais elevados de $\mathrm{P}$ e $\mathrm{K}^{+}$nas áreas sob cobertura de milheto e braquiária decorrem sobretudo da aplicação de adubos fosfatados e potássicos para o plantio de soja e do aumento nos níveis de matéria orgânica em relação ao cerrado nativo. Estudos demonstram que o aumento da matéria orgânica no solo contribui para elevação do P no solo (Canellas et al., 2003; Theodoro et al., 2003; Almeida et al., 2005) devido ao retorno das formas de $\mathrm{P}$ acumuladas na biomassa vegetal (Canellas et al., 2003) e maior complexação dos íons de Al e Fe na solução do solo (Azevedo et al., 2007).

Para os teores de $\mathrm{Ca}^{2+} \mathrm{e} \mathrm{Mg}^{2+}$, além da aplicação de calcário o efeito das plantas de coberturas também influiu nos mesmos uma vez que na área com braquiária as médias foram superiores $(p<0,05)$ em relação às encontradas na área com milheto. $\mathrm{O}$ aumento nos teores de nutrientes nas áreas sob cobertura de milheto e braquiária proporcionou acréscimo na soma de
Tabela 2. Teores de $\mathrm{P}, \mathrm{K}^{+}, \mathrm{Ca}^{2+}, \mathrm{Mg}^{2+}$ e soma de bases (SB) em um Latossolo Amarelo Distrófico com diferentes plantas de cobertura sob plantio direto na região do cerrado maranhense

\begin{tabular}{|c|c|c|c|}
\hline \multirow{2}{*}{$\begin{array}{l}\text { Profundidade } \\
\text { (m) }\end{array}$} & \multicolumn{3}{|c|}{ Plantas de cobertura } \\
\hline & Milheto & Braquiária & Cerrado nativo \\
\hline & \multicolumn{3}{|c|}{$P\left(\mathrm{mg} \mathrm{dm}^{-3}\right)$} \\
\hline $0-0,10$ & $21,33 \mathrm{aB}$ & $22,13 \mathrm{aB}$ & $0,80 \mathrm{bA}$ \\
\hline $0,10-0,20$ & $48,40 \mathrm{aA}$ & $40,77 \mathrm{aA}$ & $0,70 \mathrm{bA}$ \\
\hline \multirow[t]{2}{*}{$0,20-0,40$} & $9,20 \mathrm{aB}$ & $2,77 \mathrm{aB}$ & $0,50 \mathrm{aA}$ \\
\hline & \multicolumn{3}{|c|}{$\mathrm{K}^{+}\left(\mathrm{mg} \mathrm{dm^{-3 } )}\right.$} \\
\hline $0-0,10$ & $0,56 \mathrm{aA}$ & $0,56 \mathrm{aA}$ & $0,10 \mathrm{bA}$ \\
\hline $0,10-0,20$ & $0,17 \mathrm{aB}$ & $0,15 \mathrm{aB}$ & $0,09 \mathrm{aA}$ \\
\hline \multirow[t]{2}{*}{$0,20-0,40$} & $0,13 \mathrm{aB}$ & $0,08 \mathrm{aB}$ & $0,07 \mathrm{aA}$ \\
\hline & \multicolumn{3}{|c|}{$\mathrm{Ca}^{2+}\left(\mathrm{cmol}_{\mathrm{c}} \mathrm{dm}^{-3}\right)$} \\
\hline $0-0,10$ & $2,97 \mathrm{bA}$ & $4,93 \mathrm{aA}$ & $0,37 \mathrm{cA}$ \\
\hline $0,10-0,20$ & $1,27 \mathrm{aB}$ & $1,33 \mathrm{aB}$ & $0,33 \mathrm{bA}$ \\
\hline \multirow[t]{2}{*}{$0,20-0,40$} & $0,43 \mathrm{aC}$ & $0,50 \mathrm{aC}$ & $0,20 \mathrm{aA}$ \\
\hline & \multicolumn{3}{|c|}{$\mathrm{Mg}^{2+}\left(\mathrm{cmol}_{\mathrm{c}} \mathrm{dm}^{-3}\right)$} \\
\hline $0-0,10$ & $1,33 \mathrm{bA}$ & $1,87 \mathrm{aA}$ & $0,20 \mathrm{cA}$ \\
\hline $0,10-0,20$ & $0,50 \mathrm{aB}$ & $0,57 \mathrm{aB}$ & $0,17 \mathrm{bA}$ \\
\hline \multirow[t]{2}{*}{$0,20-0,40$} & $0,23 \mathrm{aB}$ & $0,27 \mathrm{aC}$ & $0,10 \mathrm{aA}$ \\
\hline & \multicolumn{3}{|c|}{$\mathrm{SB}\left(\mathrm{cmol}_{\mathrm{c}} \mathrm{dm}^{-3}\right)$} \\
\hline $0-0,10$ & $4,86 \mathrm{bA}$ & $7,36 \mathrm{aA}$ & $0,67 \mathrm{cA}$ \\
\hline $0,10-0,20$ & $1,94 \mathrm{aB}$ & $2,05 \mathrm{aB}$ & $0.58 \mathrm{bA}$ \\
\hline $0,20-0,40$ & $0,83 \mathrm{aC}$ & $0,85 \mathrm{aC}$ & $0,37 \mathrm{aA}$ \\
\hline
\end{tabular}

Médias seguidas de mesma letra minúscula na linha e maiúscula na coluna não diferem entre si estatisticamente pelo teste de Scott-Knott a 0,05 de probabilidade

bases (SB) quanto ao cerrado nativo devido principalmente à aplicação de corretivos e fertilizantes e ao aumento nos níveis de matéria orgânica (Tabela 3). Aumento nos teores de bases trocáveis devido à ciclagem de nutrientes via decomposição da matéria orgânica, foi verificado por Canellas et al. (2003), Theodoro et al.(2003) e Azevedo et al. (2007). Para a área sob milheto o menor teor de bases trocáveis em relação à área sob braquiária decorre provavelmente da alta capacidade de extração de nutrientes pela planta, conforme Braz et al. (2004)

Tabela 3. CTC efetiva (t), CTC potencial (T), porcentagem de saturação por bases (V) e matéria orgânica (MO) em um Latossolo Amarelo Distrófico com diferentes plantas de cobertura sob plantio direto na região do cerrado maranhense

\begin{tabular}{|c|c|c|c|}
\hline \multirow{2}{*}{$\begin{array}{l}\text { Profundidade } \\
\text { (m) }\end{array}$} & \multicolumn{3}{|c|}{ Plantas de cobertura } \\
\hline & Milheto & Braquiária & Cerrado nativo \\
\hline & \multicolumn{3}{|c|}{ CTC efetiva $\left(\mathrm{cmol}_{\mathrm{C}} \mathrm{dm}^{-3}\right)$} \\
\hline $0-0,10$ & $4,86 \mathrm{bA}$ & $7,36 \mathrm{aA}$ & $1,27 \mathrm{cA}$ \\
\hline $0,10-0,20$ & $2,47 \mathrm{aB}$ & $2,85 \mathrm{aB}$ & $1,15 \mathrm{bA}$ \\
\hline \multirow[t]{2}{*}{$0,20-0,40$} & $1,59 \mathrm{aC}$ & $1,75 \mathrm{aC}$ & $1,07 \mathrm{aA}$ \\
\hline & \multicolumn{3}{|c|}{ CTC potencial $\left(\mathrm{cmol}_{\mathrm{dm}} \mathrm{dm}^{-3}\right)$} \\
\hline $0-0,10$ & $11,24 \mathrm{bA}$ & $14,35 \mathrm{aA}$ & $5,18 \mathrm{cA}$ \\
\hline $0,10-0,20$ & $10,99 \mathrm{bA}$ & $15,06 \mathrm{aA}$ & $4,81 \mathrm{cA}$ \\
\hline \multirow[t]{2}{*}{$0,20-0,40$} & $8,09 \mathrm{bB}$ & $10,89 \mathrm{aB}$ & $4,12 \mathrm{cA}$ \\
\hline & \multicolumn{3}{|c|}{ V $(\%)$} \\
\hline $0-0,10$ & $43,10 \mathrm{bA}$ & $51,13 \mathrm{aA}$ & $13,00 \mathrm{cA}$ \\
\hline $0,10-0,20$ & $17,67 \mathrm{aB}$ & $13,47 \mathrm{aB}$ & $12,13 \mathrm{aA}$ \\
\hline $0,20-0,40$ & $10,27 \mathrm{aC}$ & $7,77 \mathrm{aC}$ & $8,97 \mathrm{aA}$ \\
\hline & \multicolumn{3}{|c|}{$\mathrm{MO}\left(\mathrm{g} \mathrm{kg}^{-1}\right)$} \\
\hline $0-0,10$ & $35,03 \mathrm{bA}$ & $48,63 \mathrm{aA}$ & $18,50 \mathrm{cA}$ \\
\hline $0,10-0,20$ & $27,53 \mathrm{bB}$ & $37,97 \mathrm{aB}$ & $17,07 \mathrm{cA}$ \\
\hline $0,20-0,40$ & $20,67 \mathrm{aC}$ & $25,50 \mathrm{aC}$ & $14,27 \mathrm{bA}$ \\
\hline
\end{tabular}

Médias seguidas de mesma letra minúscula na linha e maiúscula na coluna, não diferem entre si estatisticamente pelo teste de Scott-Knott em 0,05 de probabilidade 
A.

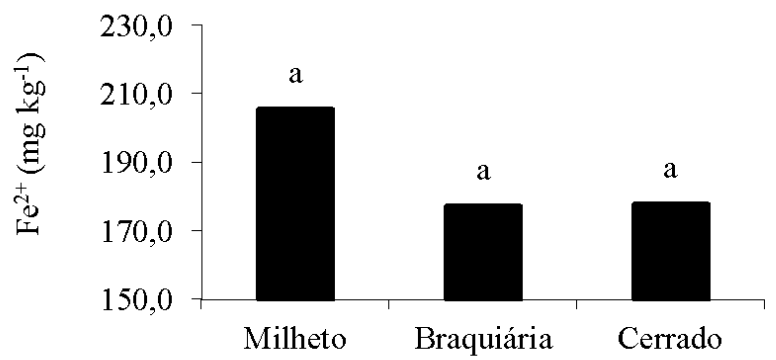

C.

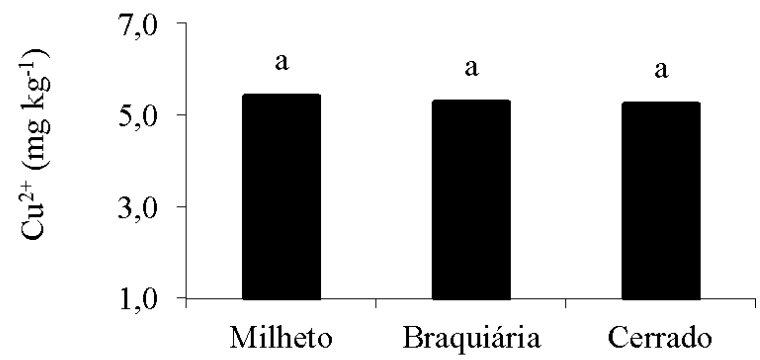

E.

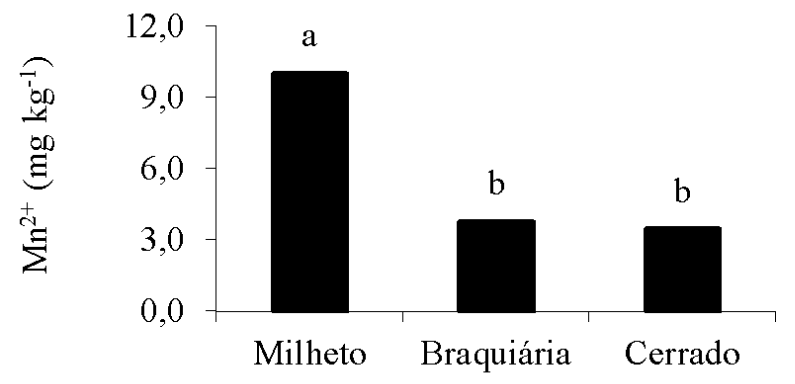

Tratamento
B.

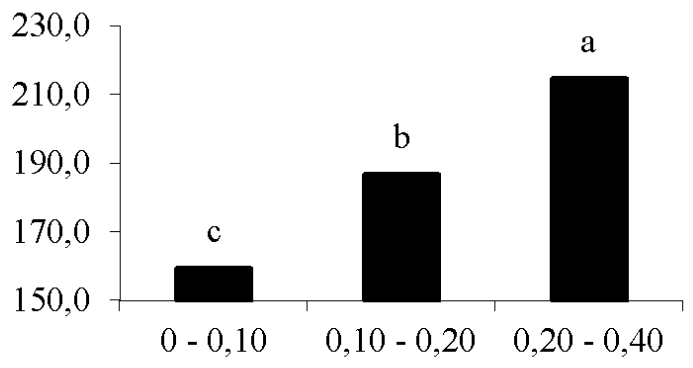

D.

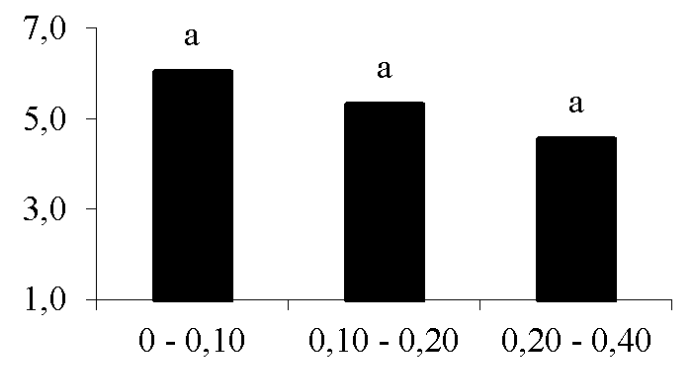

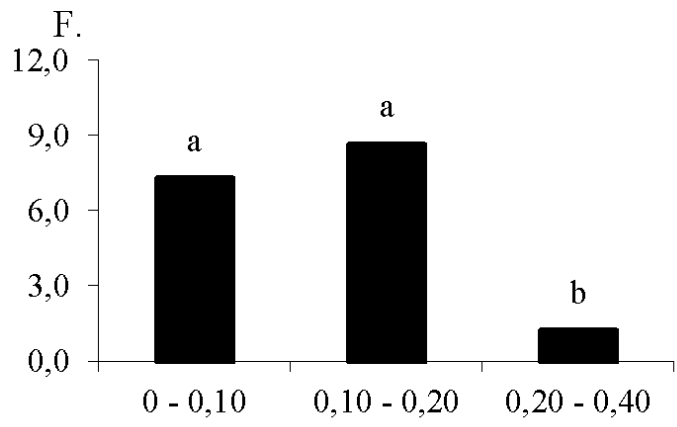

Profundidade $(\mathrm{m})$

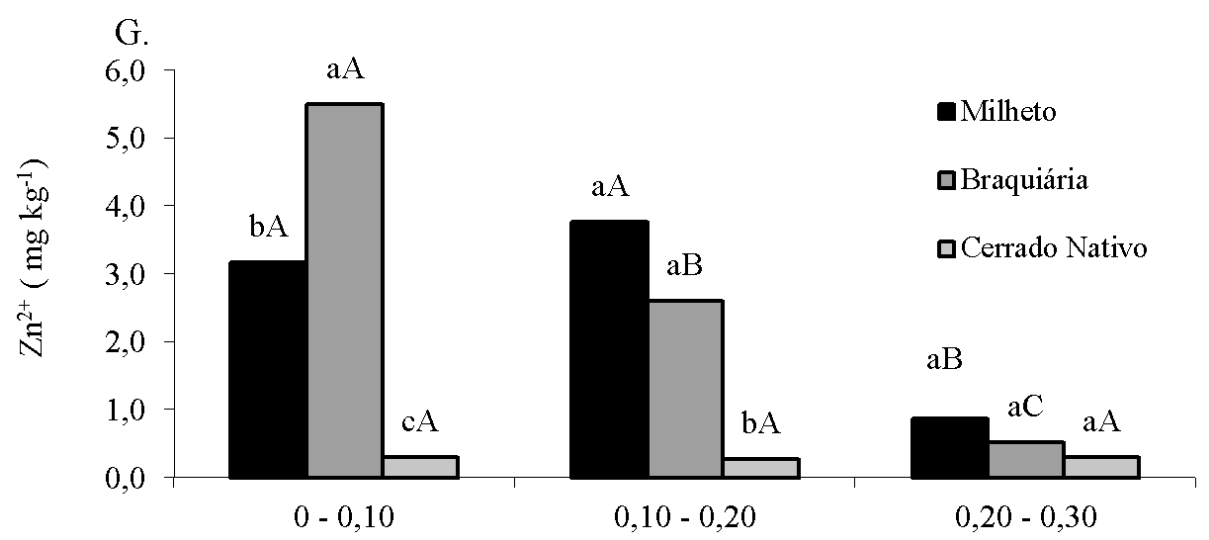

Profundidade $(\mathrm{m})$

Obs.: Letras minúsculas comparam plantas de coberturas ou profundidades entre si (Figuras A, B, C D, E, F) e as plantas de coberturas dentro de cada profundidade (Figura G). Letras maiúsculas comparam as plantas de coberturas entre profundidades (Figura $\mathrm{G}$ ) pelo teste de Skott-Knott em 0,05 de probabilidade

Figura 3. Teores de ferro, cobre, manganês e zinco em um Latossolo Amarelo Distrófico com diferentes plantas de cobertura sob plantio direto na região do cerrado maranhense 
e que ainda se encontra imobilizada nos resíduos vegetais depositados sob a superfície do solo.

Considerando a CTC efetiva e a potencial (Tabela 3 ) verificaram-se aumentos nas áreas sob cobertura com milheto e braquiária, em relação à área sob cerrado nativo, notadamente na profundidade de $0-0,10 \mathrm{~m}$. Entre as plantas de cobertura, a maior média ocorreu na área com braquiária. Este aumento decorre do acréscimo nos níveis de matéria orgânica (Tabela 3) (Canellas et al., 2003; Falleiro et al., 2003; Azevedo et al., 2007).

Aliado à aplicação de corretivos e fertilizantes, o aumento nos níveis matéria orgânica nas áreas sob coberturas de milheto e braquiária em relação ao cerrado nativo, contribui também para elevação da saturação por bases (Tabela 3 ) principalmente no solo sob braquiária. Parte deste efeito decorre da maior ciclagem dos resíduos via decomposição (Canellas et al., 2003; Theodoro et al., 2003).

Houve efeitos dos tratamentos para os teores de $\mathrm{Fe}^{2+}, \mathrm{Mn}^{2+} \mathrm{e}$ $\mathrm{Zn}^{2+}$ (Figura 3). Para os teores de $\mathrm{Fe}^{2+}$ observou-se acréscimo do nutriente em profundidade (Figura 3B) e, para o de $\mathrm{Mn}^{2+}$, na área sob cobertura de milheto (Figura 3E) e profundidade (Figura $3 \mathrm{~F}$ ). Em relação ao $\mathrm{Zn}^{2+}$ (Figura $3 \mathrm{G}$ ), os maiores teores foram registrados nas áreas sob cobertura de milheto e braquiária, sobretudo na área sob braquiária cuja média foi superior à área sob milheto $(\mathrm{p}<0,05)$.

Para a análise conjunta dos atributos dos solos avaliados sob as diferentes coberturas vegetais realizou-se a análise de componentes principais, sendo que dois componentes explicaram $79,2 \%$ da variação total $(64,7 \%$ no primeiro eixo horizontal e 14,5\% para o segundo vertical) (Figura 4). Os atributos químicos que apresentaram maior correlação com o primeiro eixo foram: $\mathrm{pH}$, com índice de correlação de $+0,98$; $\mathrm{Ca}^{2+}(+0,96), \mathrm{Mg}^{2+}(+0,96), \mathrm{SB}(+0,96)$, Efet $(-0,98), \mathrm{K}^{+}(+0,92)$, $\mathrm{Zn}^{2+}(-0,95), \mathrm{m}(0,96), \mathrm{V}(+0,98)$, POT $(+0,94) \mathrm{e} \mathrm{Al}^{3+}(-0,92)$ e para o segundo eixo asvariáveis $\mathrm{H}+\mathrm{Al}(0,90)$ e Efet $(0,85)$ (Figura 4A). Essas correlações também possibilitaram detectar diferenças entre as coberturas vegetais (milheto e braquiária) e cerrado nativo (Figura 4B).

De maneira geral pode-se visualizar o agrupamento dos atributos químicos avaliados em amostras de solo coletadas em diferentes profundidades, representados pelos pontos do diagrama em relação aos primeiro e segundo eixos, em resposta às coberturas vegetais utilizadas (Figura 4A). Foi notório o agrupamento no quadrante superior esquerdo das coberturas vegetais milheto e braquiária, coletadas nas profundidades de $0-0,10 \mathrm{~m}$ para as mesmas coberturas coletadas a $0,10-0,20$ $\mathrm{m}$ no inferior esquerdo e no inferior direito para as amostras coletadas a $0,20-0,40 \mathrm{~m}$.

Os solos coletados nas três profundidades do cerrado nativo se agruparam no quadrante superior direito e foram bem homogêneos entre si e com comportamento distinto em relação aos solos das coberturas vegetais coletados em diferentes profundidades.

Verificou-se, ainda, que as coberturas vegetais de milheto e braquiária apresentaram comportamento mais homogêneo em relação às modificações dos atributos químicos sendo que na camada de 0-0,10 $\mathrm{m}$ os atributos de maior correlação foram $\mathrm{pH}, \mathrm{Ca}^{2+}, \mathrm{Mg}^{2+}, \mathrm{SB}$, efet, $\mathrm{K}^{+}$e V. Para as amostras de solos sob
A.

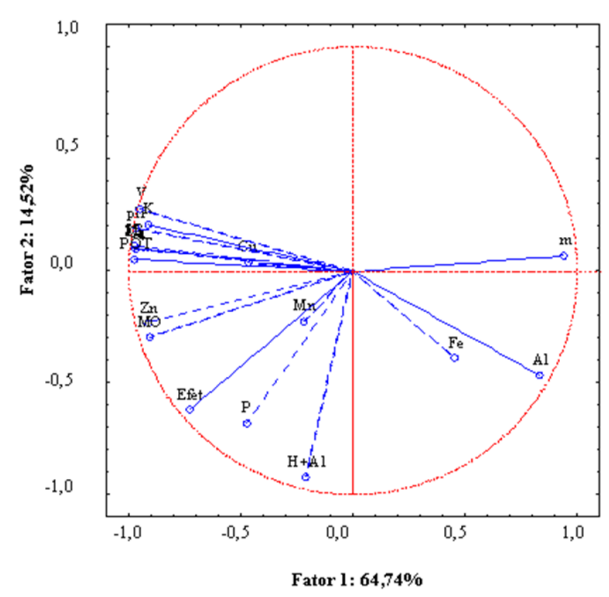

B.

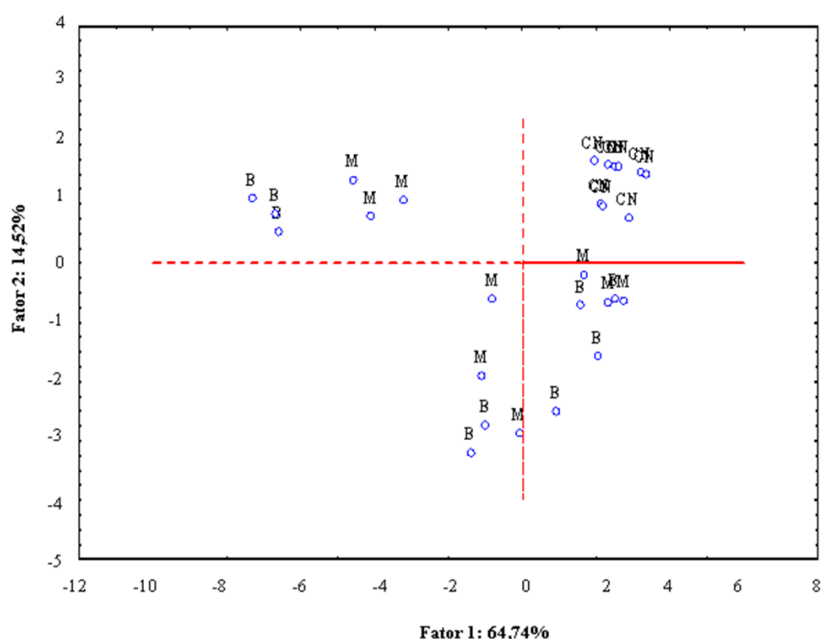

Figura 4. (A) Diagrama de ordenação das variáveis edáficas: $\mathrm{pH}$; teores de $\mathrm{K}^{+}, \mathrm{Ca}^{2+}, \mathrm{Mg}^{2+}, \mathrm{Al}^{3+}, \mathrm{Fe}^{2+}$, $\mathrm{Cu}^{2+}, \mathrm{Mn}^{2+}, \mathrm{Zn}^{2+} ; \mathrm{H}+\mathrm{Al}$; matéria orgânica (MO); soma de bases (SB); saturação por alumínio (m); capacidade de toca catiônica efetiva (Efet) e potencial (POT). (B) Tratamentos analisados: B: Braquiária; M: Milheto C: Cerrado nativo

milheto e braquiária na camada de $0,10-0,20 \mathrm{~m}$, os atributos de maior correlação foram $\mathrm{Zn}^{2+} \mathrm{e} \mathrm{MO}$ e para a camada de 0,20 $0,40 \mathrm{~m}, \mathrm{o} \mathrm{Al}^{3+}$.

\section{Conclusões}

1. Maior quantidade de resíduo vegetal foi verificada na superfície do solo da área sob cerrado nativo.

2. Os teores de nutrientes e níveis de matéria orgânica foram mais elevados nas áreas sob cobertura de milheto e braquiária, principalmente nas profundidades de $0-0,10$ e $0,10-0,20 \mathrm{~m}$.

3. A análise de componentes principais revelou que as plantas de cobertura braquiária e milheto promoveram alterações semelhantes nos atributos químicos do solo, que diferiram do cerrado nativo.

4. As variáveis edáficas que permitiram elucidar os efeitos das plantas de cobertura na camada de $0-0,10 \mathrm{~m}$ foram $\mathrm{pH}$, $\mathrm{Ca}^{2+}, \mathrm{Mg}^{2+}, \mathrm{SB}$, Efet, $\mathrm{K}^{+}$e V; na de $0,10-0,20 \mathrm{~m}, \mathrm{Zn}^{2+}$ e $\mathrm{MO}$ e na de $0,20-0,40 \mathrm{~m}, \mathrm{o} \mathrm{Al}^{3+}$. 


\section{Literatura Citada}

Almeida, J. A.; Bertol, I.; Leite, D.; Amaral, A. J.; Zoldan Jr., W. A. Propriedades químicas de um Cambissolo Húmico sob preparo convencional e semeadura direta após seis anos de cultivo. Revista Brasileira de Ciência do Solo, v.29, p.437-445, 2005.

Aratani, R. G.; Freddi, O. S.; Centurion, J. F.; Andrioli, I. Qualidade física de um Latossolo Vermelho Acriférrico sob diferentes sistemas de uso e manejo. Revista Brasileira de Ciência do Solo, v.33, p.677-687, 2009.

Azevedo, D. M. P.; Leite, L. F. C.; Teixeira Neto, M. L.; Dantas, J. S. Atributos físicos e químicos de um Latossolo Amarelo e distribuição do sistema radicular da soja sob diferentes sistemas de preparo no cerrado maranhense. Revista Ciência Agronômica, v.38, p.32-40, 2007.

Battany, M. C.; Grismer, M. E. Rainfall runoff and erosion in Napa Valley vineyards: effects of slope cover and surface roughness. Hydrology Processes, v.14, p.1289-1304, 2000.

Bortoluzzi, E. C.; Eltz, F. L. F. Efeito do manejo mecânico da palhada de aveia preta sobre a cobertura, temperatura, teor de água no solo e emergência da soja em sistema de plantio direto. Revista Brasileira de Ciência do Solo, v.24, p.449-457, 2000.

Braz, A. J. B. P.; Silveira, P. M.; Kliemann, H. J.; Zimmermann, F. J. P. Acumulação de nutrientes em folhas de milheto e dos capins braquiária e mombaça. Pesquisa Agropecuária Tropical, v.34, p.83-87, 2004.

Canellas, L. P.; Velloso, A. C. X.; Marciano, C. R.; Ramalho, J. F. G. P.; Rumjanek, V. M.; Resende, C. E.; Santos, G.A. Propriedades químicas de um Cambissolo cultivado com cana-de-açúcar, com preservação do palhiço e adição de vinhaça por longo tempo. Revista Brasileira de Ciência do Solo, v.27, p.935-944, 2003.

EMBRAPA - Empresa Brasileira de Pesquisa Agropecuária. Centro Nacional de Pesquisa de Solos. Manual de métodos de analise de solo. 2.ed. Rio de Janeiro: EMBRAPA, 1997.212p.

Espíndola, J. A. A.; Guerra, J. G. M.; Almeida, D. L. Decomposição e liberação de nutrientes acumulados em leguminosas herbáceas perenes consorciadas com bananeiras. Revista Brasileira de Ciência do Solo, v.30, p.321-328, 2006.

Falleiro, R. M.; Souza, C. M.; Silva, C. S. W.; Sediyama, C. S.; Silva, A. A.; Fagundes, J.L. Influência dos sistemas de preparo nas propriedades químicas e físicas do solo. Revista Brasileira de Ciência do Solo, v.27, p.1097-1104, 2003.

Guimarães, G. L. Efeitos de culturas de inverno e do pousio na rotação de culturas de soja e do milho em sistema de plantio direto. 108p. Ilha Solteira: Universidade Estadual Paulista, 2000.Dissertação Mestrado

Lange, A.; Carvalho, J. L. N.; Damin, V.; Cruz, J. C.; Marques, J. J. Alterações em atributos do solo decorrentes da aplicação de nitrogênio e palha em sistema semeadura direta na cultura do milho. Ciência Rural, v.36, p.460-467, 2006.

Leite, L. F. C; Mendonça, E. S., Machado, P. L. O. A., Matos, E. S. Total C and $\mathrm{N}$ storage and organic $\mathrm{C}$ pools of a RedYellow Podzolic under conventional and no tillage at the Atlantic Forest Zone, Southeastern Brazil. Australian Journal Soil Research, v.41, p.717-730, 2003.
Leite, M. H. S.; Couto, E. G.; Amorim, R. S. S.; Costa, E. L.; Maraschin, L. Perdas de solo e nutrientes num Latossolo Vermelho-Amarelo ácrico típico, com diferentes sistemas de preparo e sob chuva natural. Revista Brasileira de Ciência do Solo, v.33, p.689-699, 2009.

Moraes, R. N. S. Decomposição das palhadas de sorgo e milheto, mineralização de nutrientes e seus efeitos no solo e na cultura do milho em plantio direto. 2011. 90p. Lavras: UFLA. Dissertação Mestrado

Muñoz-Carpena, R.; Ritter, A.; Bosch, D. D.; Schaffer, B.; Potter, T. L. Summer cover crop impacts on soil percolation and nitrogen leaching from a winter corn field. Agricultural Water Management, v.95, p633-644, 2008.

Nunes, U. R.; Andrade Júnior, V. C.; Silva, E.B.; Santos, N. F.; Costa, H. A. O.; Ferreira, C. A. Covering crops straw production and common bean productivity in no-tillage system. Pesquisa Agropecuária Brasileira, v.41, p.943-948, 2006.

Odhiambo, J. J. O.; Bomke, A. A. Cover crop effects on spring soil water content and the implications for cover crop management in south coastal British Columbia. Agricultural Water Management, v.88, p.92-98, 2007.

Pavan, M. A.; Caramori, P. H.; Androcioli Filho, A.; Scholz, M. F. Manejo da cobertura do solo para formação e produção de uma lavoura cafeeira. I. Influência na fertilidade do solo. Pesquisa Agropecuária Brasileira, v.21, p.187-192, 1986.

RADAMBRASIL. Levantamento exploratório-reconhecimento de solos do Estado do Maranhão, v.1, Rio de Janeiro: EMBRAPA-SNLCS/SUDENE-DRN, 1986. 250p.

Rao, R. B.; Li, Y. C. Nitrogen mineralization of cover crop residues in calcareous gravelly soils. Commun. Soil Science Plant Analityca, v.34, p.299-313, 2003.

Sidiras, N.; Pavan, M. A. Influência do sistema de manejo do solo no seu nível de fertilidade. Revista Brasileira de Ciência do Solo, v.9, p.249-254, 1985.

Silva, M. L. N.; Curi, N.; Blancaneaux, P.; Lima, J. M.; Carvalho, A. M. Rotação adubo verde:milho e adsorção de fósforo em Latossolo Vermelho-Escuro. Pesquisa Agropecuária Brasileira, v.32, p.649-654, 1997.

Silva, T. O.; Menezes, R. S. C. Adubação orgânica da batata com esterco e, ou, Crotalaria juncea. II - Disponibilidade de N, P e K no solo ao longo do ciclo de cultivo. Revista Brasileira de Ciência do Solo, v.31, p.51-61, 2007.

Soratto, R. P.; Crusciol, C. A. C. Cátions hidrossolúveis na parte aérea de culturas anuais mediante aplicação de calcário e gesso na superfície. Revista Brasileira de Ciência do Solo, v.31, p.81-90, 2007.

Statsoft, Inc. Statistica for windows - Computer program manual. Tulsa, 2004.

Steenwerth, K.; Belina, K. M. Cover crops enhance soil organic matter, carbon dynamics and microbiological function in a vineyard agroecosystem. Applied Soil Ecology, v.40, p.359-369, 2008.

Theodoro, V. C. A.; Alvarenga, M. I. N.; Guimarães, R. J.; Souza, C. A. S. Alterações químicas em solo submetido a diferentes formas de manejo do cafeeiro. Revista Brasileira de Ciências do Solo, v.27, p.1039-1047, 2003. 
Torres, J. L. R.; Pereira, M. G.; Fabian, A. J. Produção de fitomassa por plantas de cobertura e mineralização de seus resíduos em plantio direto. Pesquisa Agropecuária Brasileira, v.43, p.421-428, 2008.
Wendling, B.; Jucksch, I.; Mendonça, E. S.; Neves, J. C. L. Carbono orgânico e estabilidade de agregados de um Latossolo Vermelho sob diferentes manejos. Pesquisa Agropecuária Brasileira, v.40, p.487-494, 2005. 